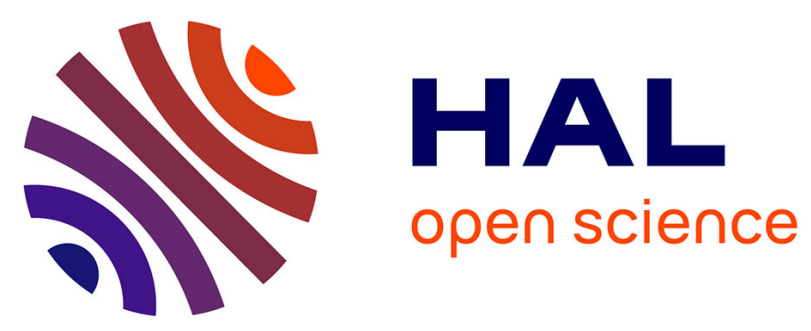

\title{
The rate-limiting step of sulfiredoxin is associated with the transfer of the $\gamma$-phosphate of ATP to the sulfinic acid of overoxidized typical 2-Cys peroxiredoxins
}

Xavier Roussel, Samia Boukhenouna, Sophie Rahuel-Clermont, Guy Branlant

\section{- To cite this version:}

Xavier Roussel, Samia Boukhenouna, Sophie Rahuel-Clermont, Guy Branlant. The rate-limiting step of sulfiredoxin is associated with the transfer of the $\gamma$-phosphate of ATP to the sulfinic acid of overoxidized typical 2-Cys peroxiredoxins. FEBS Letters, 2011, 585 (3), pp.574 - 578. 10.1016/j.febslet.2011.01.012 . hal-01682359

\section{HAL Id: hal-01682359 \\ https://hal.univ-lorraine.fr/hal-01682359}

Submitted on 15 Jan 2018

HAL is a multi-disciplinary open access archive for the deposit and dissemination of scientific research documents, whether they are published or not. The documents may come from teaching and research institutions in France or abroad, or from public or private research centers.
L'archive ouverte pluridisciplinaire HAL, est destinée au dépôt et à la diffusion de documents scientifiques de niveau recherche, publiés ou non, émanant des établissements d'enseignement et de recherche français ou étrangers, des laboratoires publics ou privés. 


\title{
THE RATE-LIMITING STEP OF SULFIREDOXIN IS ASSOCIATED WITH THE TRANSFER OF THE $\gamma$-PHOSPHATE OF ATP TO THE SULFINIC ACID OF OVEROXIDIZED TYPICAL 2-CYS PEROXIREDOXINS
}

\author{
Xavier Roussel $^{\mathrm{a}, 1}$, Samia Boukhenouna ${ }^{\mathrm{a}}$, Sophie Rahuel-Clermont ${ }^{\mathrm{a}}$ and Guy Branlant ${ }^{\mathrm{a} *}$ \\ a Unité Mixte de Recherche CNRS-Université Henri Poincaré 7214 AREMS, ARN-RNP structure- \\ fonction-maturation, Enzymologie Moléculaire et Structurale, Nancy Université, Faculté des Sciences et \\ Technologies, Bd des Aiguillettes, BP 70239, 54506 Vandœuvre-lès-Nancy Cedex, France.
}

* Corresponding author. Address: UMR CNRS-UHP 7214, Faculté des Sciences et Technologies, Bld des Aiguillettes, BP 70239, 54506 Vandœuvre-lès-Nancy Cedex, France; E-mail address: Guy.Branlant@maem.uhp-nancy.fr ; tel. +33 383684304 ; fax. +33 383684307

\footnotetext{
${ }^{1}$ Present address: Unité de Glycobiologie Structurale et Fonctionnelle, UMR8576 du CNRS, IFR147, Bâtiment C9, UST de Lille, 59655 Villeneuve d'Ascq Cedex, France
}

Abbreviations: 2-Cys-Prx: typical two-cysteine peroxiredoxin; $\mathrm{C}_{\mathrm{P}}$ : 2-Cys-Prx catalytic Cys; $\mathrm{C}_{\mathrm{R}}$ : 2-CysPrx regeneration Cys; LDH : lactate dehydrogenase ; me7Guo: methyl-7 guanosine; NTR: E.coli NADPH thioredoxin reductase; PEP : phosphoenolpyruvate; PK : pyruvate kinase ; PNP: E. coli Histagged purine nucleoside phosphorylase; $\operatorname{Prx}, \mathrm{PrxSO}_{2}$ : native and overoxidized S. cerevisiae His-tagged peroxiredoxin Tsa1; Srx: sulfiredoxin; Trx: thioredoxin. 


\begin{abstract}
The eukaryotic sulfiredoxin (Srx) catalyzes the reduction of overoxidized typical 2-Cys peroxiredoxins $\mathrm{PrxSO}_{2}$ via $\mathrm{ATP} / \mathrm{Mg}^{2+}$-dependent phosphorylation of the sulfinic acid group, followed by formation of a PrxSO-SSrx thiolsulfinate intermediate. Using real-time kinetics of wild-type and C84A Srxs, and pH-rate profiles with ATP/Mg ${ }^{2+}$ analogues, we show that the ratelimiting step of the reaction is associated with the chemical process of transfer of the $\gamma$-phosphate of ATP to the sulfinic acid, in contrast to that described by Jönsson et al. (J. Biol. Chem, 2008, 283, 22890-94). Two $\mathrm{p} K_{\text {apps }}$ of 6.2 and 7.5 were extracted from the bell-shaped pH-rate profile, corresponding to the $\gamma$-phosphate of ATP, and to an acid-base catalyst, respectively.
\end{abstract}

Keywords: Sulfiredoxin, rate-limiting step, Peroxiredoxin, ATP, sulfinic acid, phosphoryl anhydride intermediate 
Research Highlights

\section{Research Highlights}

$>$ Sulfiredoxin is rate-limited by the process of transfer of the $\gamma$-phosphate of ATP

$>$ The rate of the process is governed by ionization of the $\gamma$-phosphate of ATP

$>$ Slow rate is not expected from the chemical reactivity of substrate sulfinic acid 


\section{Introduction}

Peroxiredoxins belong to the Cys peroxidase family that reduces hydroperoxides including hydrogen peroxide $\left(\mathrm{H}_{2} \mathrm{O}_{2}\right)$ and peroxynitrite. Members of the typical eukaryotic two-Cys subfamily contain two essential cysteines. The first, termed the peroxidatic cysteine $\left(\mathrm{C}_{\mathrm{P}}\right.$, i.e. Cys48 in the case of the Saccharomyces cerevisiae 2-Cys-Prx Tsa1 and referred to as Prx in the following text), reduces hydrogen peroxide and alkyl hydroperoxide to water and alcohol, and is oxidized to a sulfenic acid (SOH). The second, the resolving cysteine $\left(\mathrm{C}_{\mathrm{R}}\right.$, i.e. Cys171) of the other Prx subunit within the homodimer attacks the sulfenic acid intermediate to form a disulfide [1-4]. This disulfide bond is reduced by thioredoxin (Trx). The peroxidatic cysteine is sensitive to overoxidation by $\mathrm{H}_{2} \mathrm{O}_{2}$ to sulfinic acid ( $\mathrm{SOOH}$ ) [4]. This overoxidation, which inactivates the enzyme, is reversed by the enzyme Sulfiredoxin (Srx) which reduces the sulfinic acid back to the sulfenic acid state [5]. Such a reversible peroxide-sensitive switch has been demonstrated to regulate the action of certain transcription factors targeting antioxidant genes in Schizosaccharomyces pombe [6].

Recently, it was shown that reduction of $\mathrm{PrxSO}_{2}$ by Srx involved a covalent intermediate linked by a thiolsulfinate bond between the essential cysteine of Srx (referred to as the catalytic cysteine and numbered 84 in the $S$. cerevisiae Srx and 99 in the human enzyme) and the $\mathrm{C}_{\mathrm{P}}$ of $\operatorname{Prx}$ [7,8]. Formation of the thiolsulfinate species requires the activation of the sulfinic acid group of $\mathrm{PrxSO}_{2}$ by transfer of the $\gamma$ phosphate of ATP in the presence of $\mathrm{Mg}^{2+}$, leading to a phosphoryl sulfinyl anhydride intermediate (Fig. 1). Reduction of the PrxSO-SSrx thiolsulfinate intermediate is completed by Trx via the formation of an intra-disulfide oxidized Srx intermediate in the case of S. cerevisiae Srx [9].

Intriguingly, the catalytic rate constant of the Srx-catalyzed reaction was found to be low with a turnover of less than $2 \min ^{-1}[8,10]$, that is limited by a step occurring before or associated with the formation of the thiolsulfinate [8]. This low rate is consistent with a role of Srx in regulation of $\mathrm{H}_{2} \mathrm{O}_{2}$ dependent signaling processes since it would enable hydrogen peroxide to convey its cellular message [11]. This raises the question of the nature of the rate-limiting process that leads to the thiolsulfinate species. Based on rapid quench/mass spectrometry/ ${ }^{18} \mathrm{O}$ exchange coupled technique, a kinetic scheme was put forward for the human Srx, in which a rapid formation of the sulfinyl phosphoryl intermediate is followed by a rate-limiting attack of Srx catalytic Cys (rate constant $1.4 \mathrm{~min}^{-1}$ ) to give the thiolsulfinate intermediate, in competition with hydrolysis (rate constant $0.35 \mathrm{~min}^{-1}$ [7]). Thus, if the proposed scheme is right, the anhydride intermediate would accumulate.

To get insight into the molecular/ mechanistic features responsible for these unexpected slow kinetics, we used both real-time enzymatic coupled systems to determine the rates of ADP and phosphate release for wild-type, C84S and C84A Srxs from S. cerevisiae, and pH-profile analysis of the rate of the 
Srx-catalyzed reaction. Taken together, our data support: i) an overall rate-limiting step for the wild-type enzyme associated with the chemical process of the ATP-dependent activation of the Prx sulfinic acid that implies a non accumulation of the sulfinyl phosphoryl anhydride intermediate. Thus, as soon as the anhydride forms, either it reacts efficiently with the catalytic Cys84 of Srx or hydrolyzes when the Cys84 is substituted by an Ala residue, and; ii) a rate of formation of the phosphorylated Prx intermediate that depends on two $\mathrm{p} K_{\text {apps }}$ of 6.2 and 7.5. The first $\mathrm{p} K_{\text {app }}$ corresponds to the $\gamma$-phosphate of ATP and the second to an acid-base catalyst which would be involved in the ADP release.

\section{Material and methods}

\subsection{Material}

Tris was obtained from VWR, $\mathrm{KCl}$ and $\mathrm{MgCl}_{2}$ from Merck, NADH and ATP- $\gamma-\mathrm{S}$ from Roche, and ATP, 7methylguanosine ( ${ }^{\mathrm{m} 7} \mathrm{Guo}$ ), phosphoenolpyruvate (PEP), lactate dehydrogenase from rabbit muscle $(\mathrm{LDH})$ and pyruvate kinase from rabbit muscle (PK) from Sigma-Aldrich.

Recombinant purine nucleoside phosphorylase from Escherichia coli (PNP), N-terminally His $6^{-}$ tagged fusion of S. cerevisiae 2-Cys-Prx Tsa1 (referred to as Prx) and S. cerevisiae Srx were prepared following the experimental procedures described previously [8]. Oxidation of $\mathrm{Cys}_{\mathrm{P}} \mathrm{C}_{\mathrm{P}}$ into the sulfinic acid state for both wild-type and C171A Prxs to form $\operatorname{PrxSO}_{2}$, and determination of the steady-state rate of the Srx-catalyzed reaction using the E. coli Trx/NADPH thioredoxin reductase (NTR) system with NADPH, were performed as described previously [8].

\section{2. $L D H / P K / N A D H$-coupled assay}

The kinetics of ADP release were followed under single turn-over conditions by the decrease of the absorbance at $340 \mathrm{~nm}$ due to the oxidation of NADH by the PK/LDH/NADH system in the presence of PEP, measured at $30{ }^{\circ} \mathrm{C}$ on an SX18MV-R stopped-flow apparatus (Applied PhotoPhysics) fitted for absorbance measurements. One syringe contained $1 \mathrm{mM}$ ATP, $3 \mathrm{mM} \mathrm{MgCl}, 1 \mathrm{mM}$ PEP, and $400 \mu \mathrm{M}$ NADH in buffer TK $(50 \mathrm{mM}$ TrisHCl, $100 \mathrm{mM} \mathrm{KCl}, \mathrm{pH}$ 7) and the other contained $80 \mu \mathrm{M} \mathrm{C171A}$ $\mathrm{PrxSO}_{2}, 20 \mathrm{U} / \mathrm{ml} \mathrm{PK}, 60 \mathrm{U} / \mathrm{ml} \mathrm{LDH}$, and $10 \mu \mathrm{M} \mathrm{Srx}$ (final concentrations after mixing). For each condition, a blank time course was recorded in the absence of Srx and the data were deducted from the corresponding data obtained in the presence of Srx. An average of three runs was recorded for each set of conditions. For wild-type Srx with C171A $\mathrm{PrxSO}_{2}$, the rate constant $k_{\text {obs }}$ was obtained by fitting blank- 
corrected absorbance traces against equation (1), in which c represents the end point and a, the amplitude of the signal. For other conditions, the rate constant $k_{\mathrm{obs}}$ was deduced by linear regression.

$$
y=a e^{-k_{o b s} t}+c
$$

The kinetic assay was validated under steady-state conditions using wild-type and $\mathrm{C} 171 \mathrm{~A} \operatorname{Prx} \mathrm{SO}_{2}$ (which behaves similarly) in the presence of Trx as reducing agent [8].

\subsection{PNP-coupled assay}

The kinetics of Pi release were followed under single turn-over conditions as described previously [8]. Briefly, the decrease of the emission fluorescence intensity associated with the phosphorolysis of

${ }^{\mathrm{m} 7}$ Guanosine $\left({ }^{\mathrm{m} 7} \mathrm{Guo}\right)$ catalyzed by PNP was recorded at $30{ }^{\circ} \mathrm{C}$ on an SX18MV-R stopped-flow apparatus fitted for fluorescence measurements, with the excitation wavelength set at $305 \mathrm{~nm}$, and the emitted light collected above $455 \mathrm{~nm}$ using a cutoff filter. One syringe contained $1 \mathrm{mM}$ ATP, $1 \mathrm{mM} \mathrm{MgCl}, 300 \mu \mathrm{M}$ ${ }^{\mathrm{m} 7}$ Guo, and $5 \mu \mathrm{M}$ PNP in buffer TK, and the other contained $80 \mu \mathrm{M} \mathrm{C171A} \mathrm{PrxSO}, 20 \mu \mathrm{M}$ PNP, and 10 $\mu \mathrm{M}$ Srx (final concentrations after mixing). For each condition, a blank time course was recorded in the absence of Srx and the data were deducted from the corresponding data obtained in the presence of Srx. For wild-type Srx with $\mathrm{C} 171 \mathrm{~A} \mathrm{PrxSO}_{2}$, the rate constant $k_{\text {obs }}$ was obtained by fitting fluorescence traces against equation (1). For other conditions, the rate constant $k_{\mathrm{obs}}$ was obtained by linear regression. For C84A Srx, the rate constant $k_{\text {obs }}$ corresponded to the initial rate constant. The fluorescence signal was calibrated independently against $\mathrm{P}_{\mathrm{i}}$ concentration.

\section{4. $p H$ dependence of the rate-limiting step of the Srx-catalyzed reaction}

The $\mathrm{pH}$-profile of the rate-limiting step of the reaction was determined by following the decrease of intrinsic tryptophan fluorescence intensity of the wild-type Prx substrate upon transitioning from the $\mathrm{SO}_{2}$ form to the disulfide form. As shown previously for the S. cerevisiae wild-type enzyme in the absence of added reductant [8], the reaction proceeds until the oxidized disulfide form of Prx is formed, whose intrinsic fluorescence signal is quenched by approximately $80 \%$ compared to the $\mathrm{PrxSO}_{2}$ substrate. The decrease of the emission fluorescence intensity was recorded at $30^{\circ} \mathrm{C}$ on an SX18MV-R stopped-flow apparatus fitted for fluorescence measurements, with excitation wavelength set at $295 \mathrm{~nm}$, and emitted light collected above $320 \mathrm{~nm}$ using a cutoff filter. One syringe contained $1 \mathrm{mM} \mathrm{ATP}, 1 \mathrm{mM} \mathrm{MgCl} 2$, and $40 \mu \mathrm{M} \operatorname{PrxSO}_{2}$, and the other contained $5 \mu \mathrm{M}$ Srx (final concentrations after mixing). Each measurement 
was carried out in $25 \mathrm{mM}$ Tricine, $100 \mathrm{mM}$ imidazole, and $75 \mathrm{mM}$ acetate buffer at an constant ionic strength of $0.1 \mathrm{M}$ over a $\mathrm{pH}$ range of 5-9 [12]. The ionic strength was verified by conductimetry and adjusted to $0.1 \mathrm{M}$ when necessary using $\mathrm{KCl}$, after calibration against $\mathrm{KCl}$ solutions.

For each condition, a blank time course was recorded in the absence of Srx and the data were deducted from the corresponding data obtained in the presence of Srx. The rate constant $k_{\mathrm{obs}}$ was obtained by fitting the fluorescence traces against equation (1). The $\mathrm{pH}$ profile of the deduced rate constants was then fitted to equation (2), in which $\mathrm{p} K_{1}$ and $\mathrm{p} K_{2}$ represent the measured apparent $\mathrm{p} K$ values, $k_{o b s m a x}$ the maximum rate constant and $\gamma k_{o b s m a x}$ the rate constant at high $\mathrm{pH}$.

$$
y=\frac{k_{o b s \max }}{1+10^{p K_{1}-p H}+10^{p H-p K_{2}}}+\frac{\gamma k_{o b s \max }}{1+10^{p H-p K_{2}}}
$$

\section{Results}

\subsection{Characterization of the rate-limiting step}

To identify which step(s) in the reaction catalyzed by the Srx from S. cerevisiae contributes to the rate of formation of the thiolsulfinate intermediate, the LDH/PK/NADH coupled assay was used to monitor the quantity of ADP released in the first step. In this assay, phosphorylation of ADP by PK in the presence of PEP leads to production of pyruvate, which is then reduced by LDH with the consumption of $\mathrm{NADH}$. Thus, the rate of ADP formation which can be monitored indirectly via the decrease in absorbance of NADH was determined under single turn-over conditions - that is, in the presence of an 8fold excess of $\mathrm{C} 171 \mathrm{~A} \mathrm{PrxSO}_{2}$ relative to wild-type Srx, and in the absence of reductant.

A control reaction lacking wild-type Srx produced a slow linear signal with rate lower than 0.05 min $^{-}$

${ }^{1}$ which was subtracted from traces collected under the same conditions in the presence of wild-type Srx. The best fit to the resulting progress curves was obtained with a monoexponential process, yielding a first order rate constant of $2.8 \pm 0.5 \mathrm{~min}^{-1}$ which was independent of wild-type Srx concentration (Fig. 2a). This rate constant is close to the rate of Pi release determined under single turnover conditions $(1.8 \pm$ $0.2 \mathrm{~min}^{-1}$ ) and to the steady-state rate constant of the reaction measured either by the NADPH/Trx/NTR coupled assay $\left(1.9 \pm 0.2 \mathrm{~min}^{-1}\right)$ [8] or by following the rate of Pi release by the PNP-coupled assay $(1.7 \pm$ $0.2 \mathrm{~min}^{-1}$ ) in the presence of excess of Trx [8]. The amplitude of the exponential process was proportional to the concentration of wild-type Srx. 


\subsection{Rates of release of ADP and phosphate by C84A and C84S Srxs}

The rates of release of $\mathrm{ADP}$ and phosphate by C84A and C84S Srxs were determined during reaction with $\mathrm{C} 171 \mathrm{~A} \mathrm{PrXSO}_{2}$ in the absence of reductant. As described previously, the release of ADP was monitored by the LDH/PK/NADH coupled assay while that of phosphate was followed by the PNP coupled assay. For C84A Srx, the time course of ADP release was linear, yielding a rate constant of 0.6 $\min ^{-1}$ (Fig. 2a). We observed a decrease in the rate of phosphate release with time, which resulted in a non-linear time course. Such a behavior remains to be explained. The rate constant for phosphate production in this case was deduced from the initial slope of the curve (Fig. 2b), giving a value of 0.7 $\mathrm{min}^{-1}$, which is similar to that of ADP release. The same experiment with C84S Srx yielded an essentially linear time course of ADP release with a rate constant of $0.1 \mathrm{~min}^{-1}$ (Fig. 2a), but no phosphate production was seen (Fig. 2b). A control experiment was carried out under the same conditions using reduced Prx instead of the overoxidized substrate and C84A Srx to evaluate the rate of background ADP and phosphate production. A rate constant of $0.05 \mathrm{~min}^{-1}$ was measured for ADP release while release of free phosphate was not observed. Thus, the phosphate which is released in the presence of C84A Srx and the substrate $\mathrm{PrxSO}_{2}$ can only derive from hydrolysis of the sulfinyl phosphoryl anhydride species formed on the overoxidized substrate. Indeed, an alternative origin for the phosphate via an ATPase activity of C84A Srx is unlikely, given that the background rate of release of ADP is only $0.05 \mathrm{~min}^{-1}$, and background production of phosphate is undetectable for this mutated Srx.

\section{3. $p H$ dependence of the rate-limiting step of the Srx-catalyzed reaction}

We did not determine the $\mathrm{pH}$-rate profiles using the PNP- or LDH/PK/NADH- coupled assays due to a potential dependence of the assays themselves on $\mathrm{pH}$. Instead, we used as a kinetic probe the attenuation of intrinsic tryptophan fluorescence of wild-type Prx upon formation of the oxidized disulfide species $\operatorname{PrxC}_{\mathrm{P}} \mathrm{S}-\mathrm{SC}_{\mathrm{R}} \mathrm{Prx}$, which is rapid and follows the rate of release of the reaction product PrxSOH [8], whose rate is itself limited by that of the activation of the Prx sulfinic acid. The experiments were carried out under sub-saturating concentrations of $\operatorname{PrxSO}_{2}\left(2 \times K_{\mathrm{M}}\right)$ to obtain a sufficient signal to noise ratio, the signal being proportional to the Srx concentration, relative to the intrinsic fluorescence of the $\mathrm{PrxSO}_{2}$. As shown in Fig. 3a, the rate of reaction exhibits a bell-shaped dependence on pH, which indicates that at least two groups are required to be in the proper ionization state for maximal catalytic activity at $\mathrm{pH}$ 7. One of these groups, characterized by a $\mathrm{p} K_{\text {app }}$ of $6.2 \pm 0.2$, must be deprotonated, whereas the other with a $\mathrm{p} K_{\text {app }}$ of $7.5 \pm 0.2$, must be protonated. When $\gamma$-S-ATP, whose $K_{\mathrm{M}}$ value is similar to that of ATP (Table 1), was used instead of ATP, the first $\mathrm{p} K_{\text {app }}$ value shifted from 6.2 to 5.5, 
while the second was determined to be of $8.0 \pm 0.2$ (Fig. 3b). Moreover, a two-fold decrease in the $k_{\text {obs }}$ max was observed (Table 1). Using $\mathrm{Mn}^{2+}$ instead of $\mathrm{Mg}^{2+}$ led i) the first $\mathrm{p} K_{\text {app }}$ value to shift from 6.2 to 5.5, and the second from 7.5 to 7.8; and ii) to similar $k_{\text {obs max }}$ (Fig. 4c) and $K_{\mathrm{M}}$ values for $\mathrm{Mn}^{2+}$ and $\mathrm{Mg}^{2+}\left(\mathrm{Table}^{2}\right.$ 1). In principle, these two $\mathrm{p} K_{\text {apps }}$ can correspond to ionizable groups on amino acids of Prx or Srx within the quaternary Srx/Prx/ATP/ $\mathrm{Mg}^{2+}$ complex or to the $\gamma$-phosphate of ATP. Because the concentration of $\mathrm{PrxSO}_{2}$ is not fully saturating, the $k_{\mathrm{obs}} v s$. $\mathrm{pH}$ curve could also include a $\operatorname{PrxSO}_{2} k_{\text {obs }} / K_{\mathrm{M}}$ contribution.

\section{Discussion}

In the present study, the rates of release of phosphate and ADP by wild-type Srxs from S. cerevisiae have been determined under single turnover conditions. In the case of wild-type Srx with $\mathrm{C} 171 \mathrm{~A} \mathrm{PrxSO}_{2}$, the rate of ADP release is similar to that of phosphate release, both determined under single turnover conditions (2.8 $\mathrm{min}^{-1}$ vs. $\left.1.8 \mathrm{~min}^{-1}\right)$ [8]. As a thiolsulfinate intermediate between Prx and wild-type Srx was shown previously to be formed at a rate of $1.7 \mathrm{~min}^{-1}$ under steady-state conditions [8], this finding supports an overall rate-limiting step for the wild type that includes all steps up to the release of ADP and thus before the formation of the thiolsulfinate intermediate.

In a previous study, a stoichiometry of one mole of phosphate released per mole of wild-type Srx was observed upon formation of the thiolsulfinate intermediate in the absence of added reductant [8]. Combined with the above result, this demonstrates that hydrolysis of the sulfinyl phosphate does not occur, nor is detectable under the experimental conditions used. This finding implies that each sulfinyl phosphate anhydride Prx intermediate which is formed proceeds to the thiolsulfinate Prx/Srx species at a much higher intrinsic rate than the $1.4 \mathrm{~min}^{-1}$ reported [7] and does not accumulate. The reason why the Prx intermediate does not hydrolyze but forms a thiolsulfinate Prx/Srx intermediate is the higher chemical reactivity of the $\operatorname{Srx}$ Cys84 (whose $\mathrm{p} K_{\text {app }}$ is $7.4^{1}$ - as also shown for the human Srx [10]) towards the sulfinyl phosphate anhydride intermediate, relative to a non activated water molecule, a reactivity which is further favored by an entropic effect due to the relative positioning of the anhydride intermediate and Cys84 within the Prx/Srx complex.

To get deeper into the mechanism of phosphate transfer from ATP to the sulfinate group of $\mathrm{PrxSO}_{2}$, we performed a kinetic analysis of ADP release rates using C84A and C84S mutants of Srx. As deduced from Fig. 2, the rates of release of ADP and phosphate with C84A Srx are in the same range and

significantly higher (0.6-0.7 $\left.\mathrm{min}^{-1}\right)$ than the background. Therefore, formation of a phosphoryl-Cys intermediate on the Cys 84 of wild-type Srx can be excluded, in contrast to that suggested by Jeong and co-workers [13]. It is also in accord with structural data obtained on the human enzyme [14]. The fact that both rates are similar and the time course, in particular of ADP release, is linear indicates no 
accumulation of the sulfinyl phosphoryl anhydride intermediate. Therefore, the 'intrinsic' rate of hydrolysis of this intermediate is likely significantly higher than $0.6 \mathrm{~min}^{-1}$. Given the difference of $\mathrm{p} K_{\mathrm{a}}$ between sulfinic and acetic acid (1.9 vs. 4.3, respectively), this result is consistent with the rate of hydrolysis of a phosphoryl carboxylic anhydride like acetyl phosphate, $\left(0.004\right.$ and $0.01 \mathrm{~min}^{-1}$ at $\mathrm{pH} 7.7$ in the absence and presence of $100 \mathrm{mM}$ of $\mathrm{Mg}^{2+}$, respectively [15]). Due to the regeneration of the substrate $\mathrm{PrxSO}_{2}$, a futile cycle occurs that consumes ATP with linear, steady state kinetics, and an amplitude that is only limited by ATP and $\mathrm{Mg}^{2+}$ concentration, contrary to the wild-type mechanism that corresponds to single-turnover kinetics, with amplitude limited by the concentration of Srx.

The decrease in the rates of ADP and phosphate release for C84A Srx relative to wild type when using C171 $\mathrm{ArxSO}_{2}$ as a substrate could be caused by a change in the environment near the $\gamma$-phosphate of ATP due to the substitution of Cys by Ala. In the case of C84S Srx, the rate of release of ADP is 0.1 $\min ^{-1}$, which is only two-fold higher than the background rate, and 28 and 6-fold lower than the rate observed with wild-type and C84A Srxs, respectively. These data suggested that for as yet undetermined structural reasons which is likely related to the high flexibility of the active site of Srx, the quaternary $\mathrm{PrxSO}_{2} / \mathrm{ATP} / \mathrm{Mg}^{2+} / \mathrm{C} 84 \mathrm{~S}$ Srx complex is not competent to transfer the $\gamma$-phosphate of ATP to PrxSO ${ }_{2}$. The fact that no phosphate release was observed supports this interpretation.

The $\mathrm{pH}$ dependence of the rate of formation of the sulfinyl phosphoryl anhydride shows a bellshaped profile from which two $\mathrm{p} K_{\text {apps }}$ of 6.2 and 7.5 were extracted. Based on the above demonstration that phosphate transfer does not depend on Cys84, the first ionization of $\mathrm{p} K_{\text {app }} 6.2$ may be attributed to either the sulfinate group of $\mathrm{PrxSO}_{2}$, or to the $\gamma$-phosphate group of ATP. Attribution of the ionization to the $\gamma$-phosphate was established from the observation that the first $\mathrm{p} K_{\text {app }}$ decreased when ATP- $\gamma-\mathrm{S}$ and $\mathrm{Mn}^{2+}$ were used instead of ATP [16,17] and $\mathrm{Mg}^{2+}[18]$, respectively. Taken together, the data clearly demonstrate: i) a rate-limiting step associated with the chemical process leading to the transfer of the $\gamma$ phosphate from ATP to the sulfinic acid; and ii) the $\gamma$-phosphate of ATP as the group with a $\mathrm{p} K_{\text {app }}$ of 6.2, and thus excludes the sulfinic acid whose $\mathrm{p} K_{\text {app }}$ in solution is $~ 2$ [19].

The nature of the second $\mathrm{p} K_{\text {app }}$ remains to be determined. The difficulty with this measurement stems partly from the overall low rate of turnover of Srx, which precludes kinetic comparison of the effects of different mutations introduced into the active site. The transfer of the $\gamma$-phosphate involves the release of ADP which is likely to be assisted by an acid catalyst. A candidate residue deduced from the inspection of the crystal structure of human Srx in complex with ATP- $\mathrm{Mg}^{2+}$ is His100 [14], which corresponds to His85 in the S. cerevisiae Srx. Indeed, substitution of this His by Asn either in the S. cerevisiae ${ }^{1}$ or human [13] enzymes leads to a complete loss of activity, with a $k_{\text {cat }}$ value approximately equal to that of background (e.g. a 20-fold decrease at least). 
From a chemical point of view, the rate of the phosphotransferase activity was expected to be rapid. Indeed, a sulfinic acid has a $\mathrm{p} K_{\text {app }}$ of $\sim 2$ and is a better nucleophile than a carboxylate group [19]. However, the measured $k_{\text {obs max }}$ value is low $\left(2 \mathrm{~min}^{-1}\right)$. The structural/molecular factors which would be responsible for this low $k_{\text {obs max }}$ value remains to be elucidated. Nevertheless, the rate of formation of the sulfinyl phosphoryl anhydride intermediate, although low, is sufficient to repair the overoxidized Prx as shown by in vivo experiments $[5,10]$. This fact could explain why no additional evolutionary pressure has occurred to force the sulfinic acid to reveal its 'intrinsic' chemical reactivity.

\section{Footnote}

${ }^{1} \mathrm{~S}$. Boukhenouna, personal communication

\section{References}

[1] Kang, S.W., Baines, I.C., and Rhee, S.G. (1998) Characterization of a mammalian peroxiredoxin that contains one conserved cysteine. J. Biol. Chem. 273, 6303-6311.

[2] Kong, W., Shiota, S., Shi, Y., Nakayama, H., and Nakayama, K. (2000) A novel peroxiredoxin of the plant Sedum lineare is a homologue of Escherichia coli bacterioferritin co-migratory protein (Bcp). Biochem. J. 351, 107-114.

[3] Baker, L.M., and Poole, L.B. (2003) Catalytic mechanism of thiol peroxidase from Escherichia coli. Sulfenic acid formation and overoxidation of essential CYS61. J. Biol. Chem. 278, 92039211.

[4] Wood, Z.A., Schroder, E., Robin Harris, J., and Poole, L.B. (2003) Structure, mechanism and regulation of peroxiredoxins. Trends Biochem. Sci. 28, 32-40.

[5] Biteau, B., Labarre, J., and Toledano, M.B. (2003) ATP-dependent reduction of cysteinesulphinic acid by $S$. cerevisiae sulphiredoxin. Nature 425, 980-984.

[6] Vivancos, A.P., Castillo, E.A., Biteau, B., Nicot, C., Ayte, J., Toledano, M.B., and Hidalgo, E. (2005) A cysteine-sulfinic acid in peroxiredoxin regulates $\mathrm{H}_{2} \mathrm{O}_{2}$-sensing by the antioxidant Pap1 pathway. Proc. Natl. Acad. Sci. U. S. A. 102, 8875-8880.

[7] Jonsson, T.J., Tsang, A.W., Lowther, W.T., and Furdui, C.M. (2008) Identification of intact protein thiosulfinate intermediate in the reduction of cysteine sulfinic acid in peroxiredoxin by human sulfiredoxin. J. Biol. Chem. 283, 22890-22894.

[8] Roussel, X., Bechade, G., Kriznik, A., Van Dorsselaer, A., Sanglier-Cianferani, S., Branlant, G., and Rahuel-Clermont, S. (2008) Evidence for the formation of a covalent thiosulfinate intermediate with peroxiredoxin in the catalytic mechanism of sulfiredoxin. J. Biol. Chem. 283, 22371-22382.

[9] Roussel, X., Kriznik, A., Richard, C., Rahuel-Clermont, S., and Branlant, G. (2009) Catalytic mechanism of Sulfiredoxin from Saccharomyces cerevisiae passes through an oxidized disulfide sulfiredoxin intermediate that is reduced by thioredoxin. J. Biol. Chem. 284, 33048-33055. 
[10] Chang, T.-S., Jeong, W., Woo, H.A., Lee, S.M., Park, S., and Rhee, S.G. (2004) Characterization of Mammalian Sulfiredoxin and Its Reactivation of Hyperoxidized Peroxiredoxin through Reduction of Cysteine Sulfinic Acid in the Active Site to Cysteine. J. Biol. Chem. 279, 5099451001.

[11] Hall, A., Karplus, P.A., and Poole, L.B. (2009) Typical 2-Cys peroxiredoxins--structures, mechanisms and functions. Febs J. 276, 2469-2477.

[12] Ellis, K., and Morrison, J. (1982) Buffers of constant ionic strength for studying pH-dependent processes. Methods Enzymol. 87, 405-426.

[13] Jeong, W., Park, S.J., Chang, T.S., Lee, D.Y., and Rhee, S.G. (2006) Molecular mechanism of the reduction of cysteine sulfinic acid of peroxiredoxin to cysteine by mammalian sulfiredoxin. J. Biol. Chem. 281, 14400-14407.

[14] Jonsson, T.J., Murray, M.S., Johnson, L.C., and Lowther, W.T. (2008) Reduction of cysteine sulfinic acid in peroxiredoxin by sulfiredoxin proceeds directly through a sulfinic phosphoryl ester intermediate. J. Biol. Chem. 283, 23846-23851.

[15] Koshland, D.E. (1952) Effect of Catalysts on the Hydrolysis of Acetyl Phosphate. Nucleophilic Displacement Mechanisms in Enzymatic Reactions1. J. Am. Chem. Soc. 74, 2286-2292.

[16] Pecoraro, V.L., Hermes, J.D., and Cleland, W.W. (1984) Stability constants of $\mathrm{Mg}^{2+}$ and Cd ${ }^{2+}$ complexes of adenine nucleotides and thionucleotides and rate constants for formation and dissociation of MgATP and MgADP. Biochemistry 23, 5262-5271.

[17] Frey, P.A., and Sammons, R.D. (1985) Bond order and charge localization in nucleoside phosphorothioates. Science 228, 541-545.

[18] Sigel, H. (1987) Isomeric equilibria in complexes of adenosine 5'-triphosphate with divalent metal ions. Solution structures of $\mathrm{M}(\mathrm{ATP})_{2}$ - complexes. Eur. J. Biochem. 165, 65-72.

[19] Reddie, K.G., and Carroll, K.S. (2008) Expanding the functional diversity of proteins through cysteine oxidation. Curr. Opin. Chem. Biol. 12, 746-754.

\section{Acknowledgements}

This work was supported in part by the CNRS, the University of Nancy I, the Institut Fédératif de Recherche 111 Bioingénierie, and by the French Agence Nationale de la Recherche (program ANR-06BLAN-0369). X. Roussel and S. Boukhenouma was and is supported, respectively by the French Research Ministry. We thank I. Artaud and K. Weissman for helpful discussions and A. Kriznik and J. Charbonnel for excellent technical assistance. 
Figure legends

Figure 1. Catalytic mechanism of the formation of the thiolsulfinate intermediate of Srx. P: Prx; S: Srx.

Figure 2. Single turnover kinetics of the reaction catalyzed by Srx with $\mathrm{C171A} \mathrm{PrxSO}_{2}$. Reactions of $80 \mu \mathrm{M}$ C171A PrxSO 2 with $10 \mu \mathrm{M}$ wild-type (black), C84S (grey) and C84A (light grey) Srxs in the presence of $1 \mathrm{mM} \mathrm{ATP} / \mathrm{MgCl}_{2}$ were carried out in buffer TK at $30{ }^{\circ} \mathrm{C}$. (a) The reaction was monitored by ADP release by the LDH/PK/NADH coupled assay (see section 2.2). (b) The reaction was monitored by the PNP coupled assay (see section 2.3). The progress curves are corrected from data collected in the absence of Srx.

Figure 3. Representative pH dependence of the rate-limiting step of the Srx-catalyzed reaction. Reaction of $40 \mu \mathrm{M}$ wild-type $\mathrm{PrxSO}_{2}$ and $5 \mu \mathrm{M}$ wild-type $\mathrm{Srx}$ was followed at $30^{\circ} \mathrm{C}$ by the attenuation of the intrinsic tryptophan fluorescence of $\mathrm{PrxSO}_{2}$ (see section 2.4) in the presence of $1 \mathrm{mM}$ ATP, $1 \mathrm{mM}$ $\mathrm{MgCl}_{2}$ (a), $1 \mathrm{mM}$ ATP- $\gamma-\mathrm{S}$ and $1 \mathrm{mM} \mathrm{MgCl}$ (b) or $1 \mathrm{mM}$ ATP, $1 \mathrm{mM} \mathrm{MnCl}_{2}$ (c). Each measurement was carried out in $25 \mathrm{mM}$ tricine, $100 \mathrm{mM}$ imidazole, and $75 \mathrm{mM}$ acetate buffer at a constant ionic strength of $0.1 \mathrm{M}$. Apparent $\mathrm{p} K_{1}$ and $\mathrm{p} K_{2}$ values of $6.2 \pm 0.2$ and $7.5 \pm 0.2$ (a); $5.5 \pm 0.1$ and $8.0 \pm 0.2$ (b); and $5.5 \pm$ 0.1 and $7.8 \pm 0.2(\mathrm{c})$ were deduced from the best fit (solid line) of the experimental points $(\bullet)$ against equation (2). Results are expressed as the average of two independent determinations ( \pm SD). 
Table 1. Kinetic parameters of wild-type Srx for the co substrates ATP and $\mathrm{Mg}^{2+}$, and for the co substrate analogs $\gamma$-thio-ATP and $\mathrm{Mn}^{2+}$.

The Michaelis constants were determined from activity measurement performed using the Trx/NTR coupled assay in TK buffer $\mathrm{pH} 7$ at $30^{\circ} \mathrm{C}$ by following the consumption of NADPH at $340 \mathrm{~nm}$. Reaction of $50 \mu \mathrm{M} \operatorname{PrxSO}_{2}, 200 \mu \mathrm{M}$ NADPH, $50 \mu \mathrm{M}$ Trx, $0.5 \mu \mathrm{M}$ NTR and $1 \mathrm{mM} \mathrm{MgCl} \mathrm{Mg}_{2}$ or $1 \mathrm{mM}$ ATP with variable concentrations of the indicated co substrate, were initiated by adding $10 \mu \mathrm{M}$ Srx.

\begin{tabular}{lllll}
\cline { 2 - 4 } & ATP & $\gamma$-thio-ATP & $\mathrm{MgCl}_{2}$ & $\mathrm{MnCl}_{2}$ \\
\hline$K_{\mathrm{M}}(\mu \mathrm{M})$ & $125 \pm 50$ & $130 \pm 30$ & $250 \pm 60$ & $230 \pm 70$ \\
\hline$k_{\text {obs max }}\left(\mathrm{min}^{-1}\right)$ & $1.83 \pm 0.18$ & $0.96 \pm 0.04$ & $1.82 \pm 0.13$ & $1.75 \pm 0.15$ \\
\hline
\end{tabular}




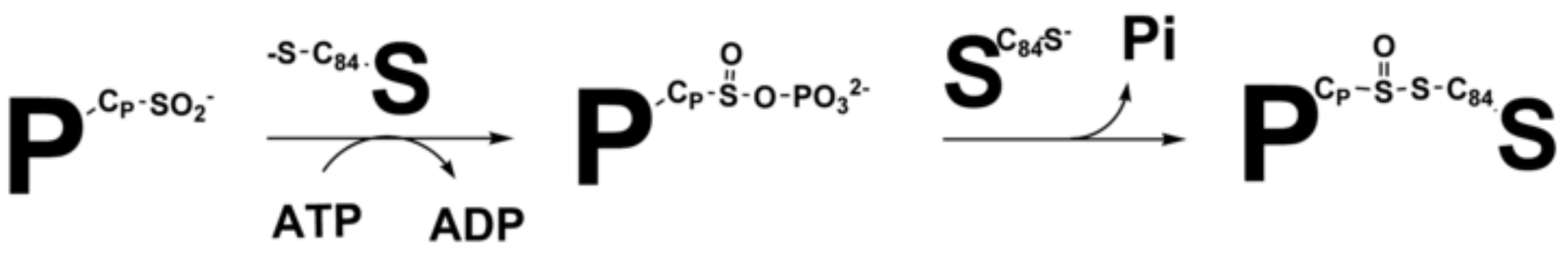



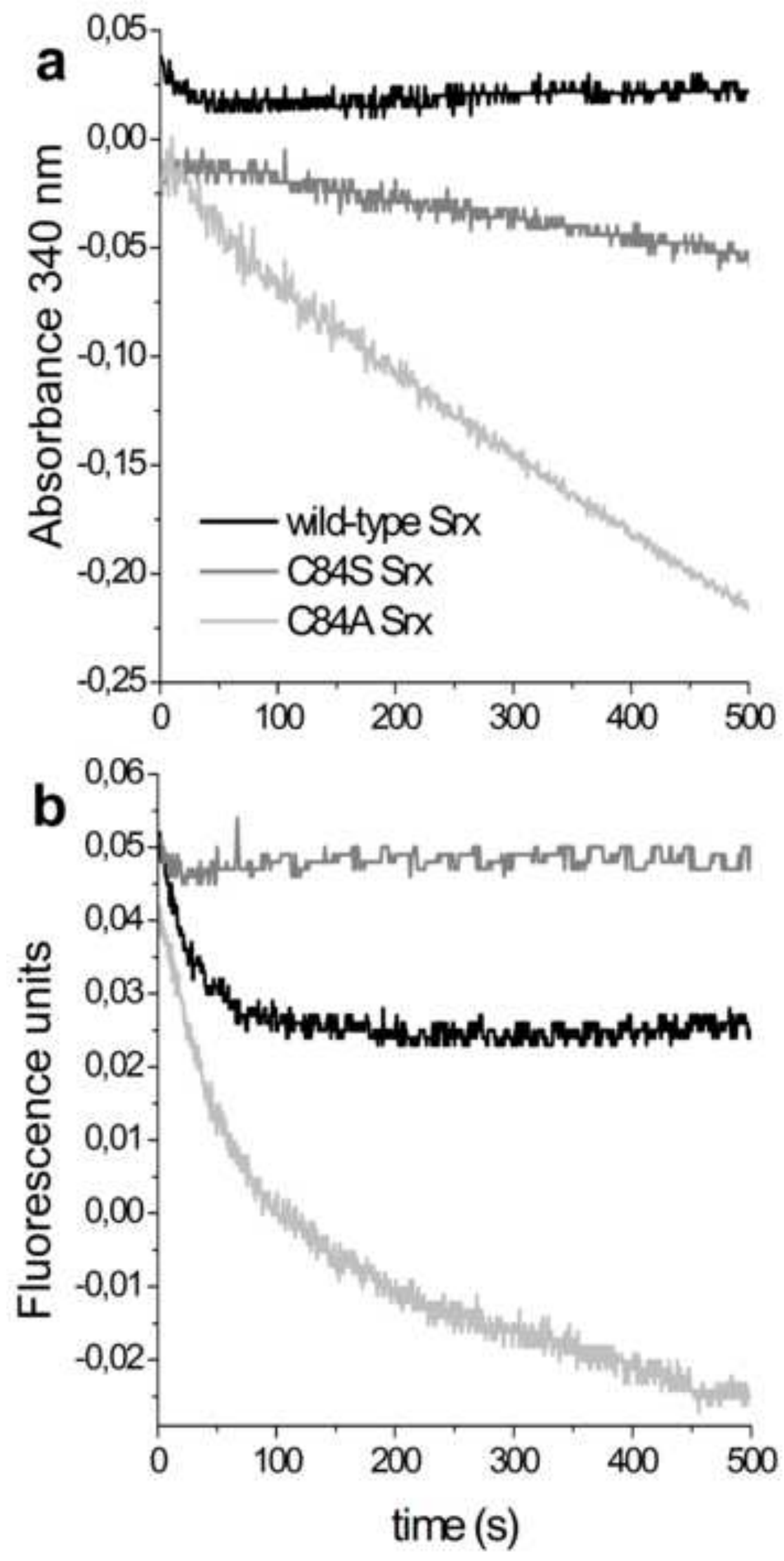
Figure3

Click here to download high resolution image

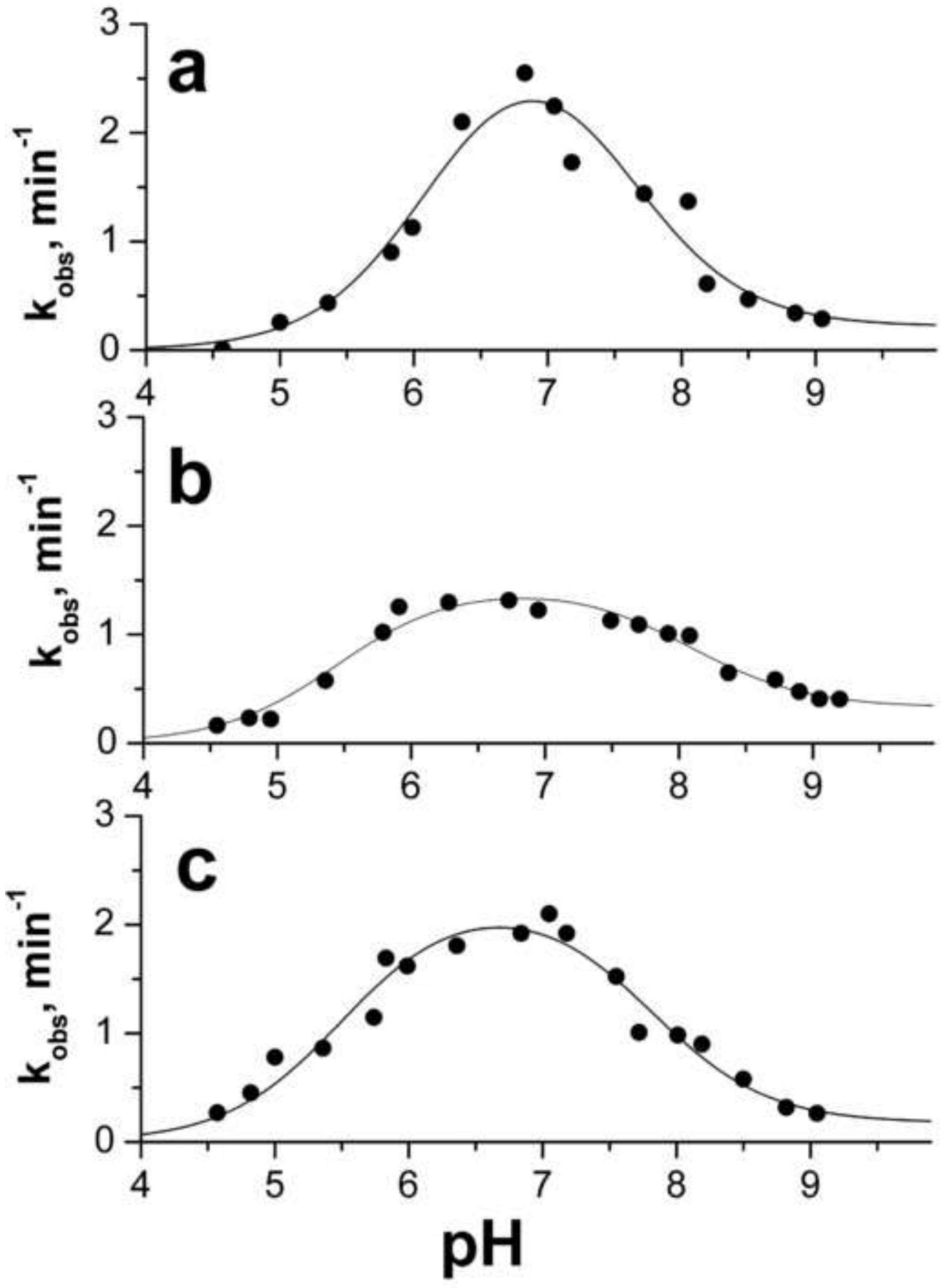



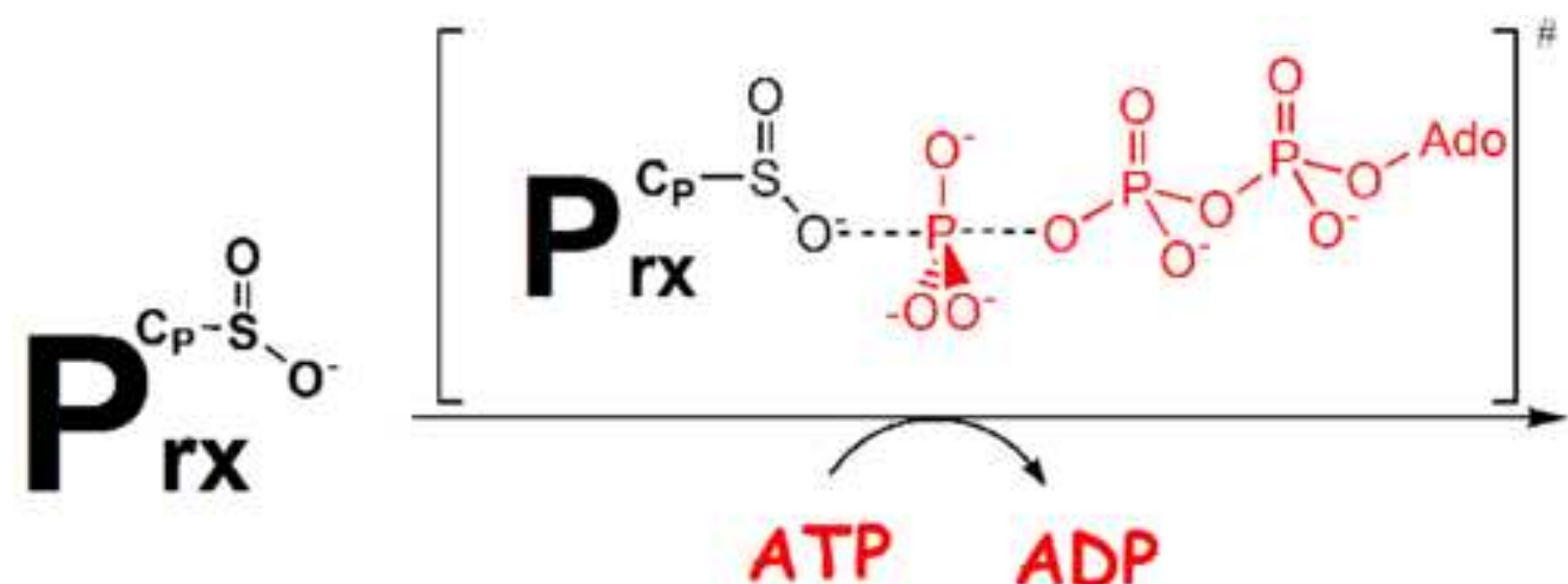

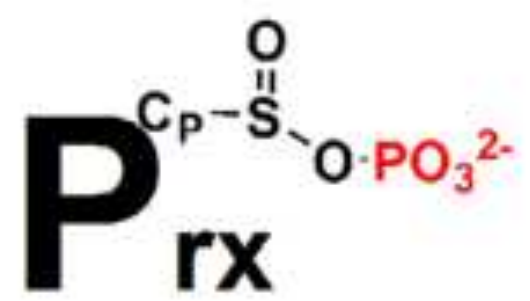

$\mathrm{k}=2 \mathrm{~min}^{-1}$
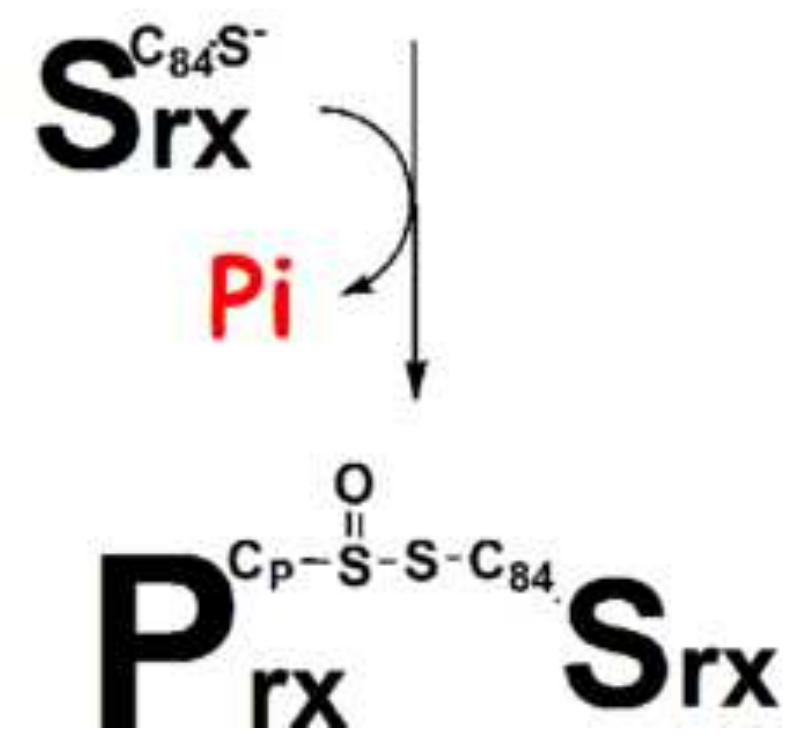